\title{
A Resolution of the Sylvester-Gallai Problem of J.-P. Serre
}

\author{
L. M. Kelly \\ Department of Mathematics, Michigan State University, East Lansing, MI 48824-1027
}

\section{Introduction}

Almost from the beginning of the study of algebraic curves in which imaginary points were admitted, it has been known that a general plane cubic curve has nine inflection points which form a rather curious configuration, i.e., the line joining any two of them passes through a third.

It was undoubtedly this configuration which prompted Sylvester to challenge his contemporaries in 1893 [9] to show that it was impossible to have a non-linear finite set of points in real space exhibiting this same curious characteristic. His contemporaries did not satisfactorily resolve the issue and it is not clear whether Sylvester himself knew of a proof.

In any event the problem seems to have passed into limbo until it was resurrected in 1933 by Erdös in Hungary and by Motzkin [6] in Israel. Erdös' countryman, T. Gallai, produced a very simple and elementary argument affirming the Sylvester conjecture.

Finite point sets in projective or affine spaces which are such that the line joining any two points contains at least one more are now called Sylvester-Gallai configurations. This definition admits finite linear sets as SGC's and Gallai's proof permits us to assert that in real space SGC's are confined to the line. In fact his argument and many that followed it prove the stronger result.

Theorem. SGC's in any ordered projective or affine space are confined to the line.

Since any finite projective or affine space of any dimension is an SGC, it is clear that it is not the incidence of configurational requirements (Pappus, etc.) that inhibit the existence of nonlinear SGC's in real space. It is clear that "order implies Sylvester" but whether "Sylvester implies order" is an intriguing open question which adds interest to the next observation. 


\section{The Serre Problem}

SGC's in complex space are. of course, not confined to the line, but in 1966 J.-P. Serre [8] wondered if possibly they might be confined to the plane. It is easy to construct nonlinear SGC's of cardinality $3 k$ for any $k \geq 3$ in the complex plane but no SGC spanning complex 3-space had been found. The reason is simple. There are none as we now show.

Theorem. SGC's in complex space are confined to the plane.

The proof depends on two lemmas, one easily proved, the other a corollary of a probably deep theorem recently uncovered in algebraic geometry. The lemma from algebraic geometry appears in a paper by $F$. Hirzebruch [2] and is a corollary of an inequality relating the Chern numbers of an algebraic surface of general type. The inequality is known as the Miyaoka-Yau inequality [5], [10]. Hirzebruch studies "arrangements of lines" in the complex plane and associates with each such arrangement algebraic surfaces, calculates their Chern numbers and applies the Miyaoka-Yau inequality. If exactly $j$ lines of an arrangement pass through a point, the point is a $j$-vertex of the arrangement and the number of $j$-vertices is denoted $t_{j}$.

One of the early proofs of the Sylvester theorem flows easily from an inequality due to Melchior [4] for real arrangements, namely, $t_{2} \geq 3+t_{4}+2 t_{5}+$ $3 t_{6}+\ldots$. This inequality is an easy consequence of the Euler formula applied to the partition of the real plane by the lines of the arrangement. It is instantly obvious, of course, that $t_{2} \geq 3$ which is the dual form of the Sylvester theorem.

This inequality is clearly not valid in the complex plane and the Hirzebruch lemma provides us with the analogue in this setting. For an arrangement of $k$ lines in the complex plane, the Miyaoka-Yau inequality implies, according to Hirzebruch, that if $t_{k}=t_{k-1}=0$, then

$$
t_{2}+t_{3} \geq k+t_{5}+2 t_{6}+3 t_{7}+\ldots
$$

Dualizing, we have as an immediate consequence that any nonlinear SGC in the complex plane has a 3-line which is the key fact we need for our proof that SGC's in complex space are confined to the plane.

We might observe, in passing, that the Hirzebruch inequality permits a Sylvester-like assertion in complex space, i.e., it is impossible to have a finite nonlinear set of points in complex space such that the line joining any two points passes through at least two more of the points.

The second lemma needed for the proof of theorem 1 is quite easy to prove and asserts that in the complex plane an SGC cannot be a subset of three concurrent lines. We emphasize the concurrency since all the examples (alluded to above) of SGC's in complex plane of cardinality a multiple of 3 are subsets of 3 lines but they are not concurrent. We prove a bit more.

Lemma 2. If a nonlinear SGC in a pappian affine plane is a subset of 3 concurrent lines, then the associated field has nonzero characteristic. 


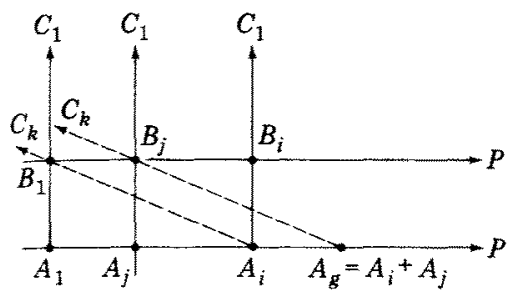

Proof. Suppose in the plane $\pi$ the SGC $S$ is a subset of the three lines $l_{1}, l_{2}, l_{3}$ meeting in $P$. We may assume a coordinate system such that $l_{1}$ is the $x$-axis, $P$ the ideal point on $l_{1}, l_{2}$ is the line $y=1$ and $l_{3}$ is the ideal line.

Suppose $A_{1}, A_{2}, \ldots, A_{j}$ are the points of $S-P$ on $l_{1}, B_{1}, B_{2}, \ldots, B_{j}$ the points on $l_{2}$ and $C_{1}, C_{2}, \ldots, C_{j}$ the points on $l_{3}$. It is easily seen that the cardinality of each of these sets is the same.

We may further assume that the line $A_{1} B_{1}$ is the $y$-axis and $C_{1}$ is the ideal point on this line. $A_{1}$ then has coordinates $(0,0)$ and the coordinates of $A_{i}$ may be denoted $\left(a_{i}, 0\right)$. There is no loss of generality in assuming that the line $C_{1} A_{\text {, }}$ intersects $l_{2}$ in $B_{i}$.

Now the line $B_{1} A_{i}$ goes through some point $C_{k}$ and the line $C_{k} B_{J}$ in turn goes through some $A_{g}$ which by the usual addition scheme in affine coordinates is the point $A_{i}+A_{j}$.

Thus the points of $S-P$ on the $x$-axis are closed under addition and hence form an additive subgroup of the additive group of the field. This is only possible if the characteristic of the field is nonzero.

We can now proceed to a proof of our theorem.

Proof of the Theorem. Suppose $S$ a nonplanar SGC in complex 3-space, $P \in S$ and $\pi$ a plane not containing $P$. Let $S^{*}$ be the central projection of $S-P$ onto $\pi$ from $P . S^{*}$ is easily seen to be a nonlinear SGC in $\pi$ and thus by the Hirzebruch inequality must define a 3-line $l$. But then the intersection of the plane containing $l$ and $P$ with $S$ is a nonlinear SGC in complex space lying on three concurrent lines which is impossible.

\section{Remarks}

1. As satisfying as this proof might be to devotees of the theory of characteristic classes, a confirmed configurationalist cannot but feel that Hirzebruch's key inequality should be susceptible to a much more direct derivation.

Conjecture. If in real 4-space, each two lines of a finite family, $\Sigma$, spans a 3-space which contains at least one more line of $\Sigma$, then $\Sigma$ is contained in a 3-space.

If true, this could replace the Hirzebruch inequality for our present purposes.

2. The inequality $c_{1}^{2} \leq 3 c_{2}$ relating the Chern numbers of a surface of general type has an interesting history. S. Kobayashi traces its origins and development in some detail in [3]. 
3. The Sylvester-Gallai configuration is intimately related to another classical configuration of considerable historical interest, namely, the so-called desmic configuration. Implications of the present result in this direction will be the subject of a forthcoming note.

4. Hirzebruch has improved his inequality in [1]. The improved version is

$$
t_{2}+\frac{3}{4} t_{3} \geq k+\sum_{r \geq 5}(2 r-9) t_{r}
$$

provided $t_{k}=t_{k-1}=t_{k-2}=0$. See also [1, appendix] and [7].

\section{References}

1. F. Hirzebruch, Singularities of Algebraic Surfaces and Characteristic Numbers, Max Planck Inst. für Mathematik, Bonn, 1984.

2. F. Hirzebruch, Arrangements of lines and algebraic surfaces, in Arithmetic and Geometry (Papers dedicated to I. R. Shafarevich), vol. 2, 113-140, Birkhäuser, 1983.

3. S. Kobayashi, Recent results in complex differential geometry, Jber. d. Dt. Math-Verein 83 (1981), 147-158.

4. E. Melchior, Über Vielseite der projective Ebene, Deutsche Math. 5 (1940), $461-475$.

5. Y. Miyaoka, On the Chern class numbers of surfaces of general type, Inv. Math. 42 (1977), 225-237.

6. T. S. Motzkin. The lines and planes connecting the points of a finite set, Trans. Am. Math. Soc. 70 (1951), $451-464$.

7. F. Sakai, Semi-stable curves on algebraic surfaces and logarithmic pluricanonical maps, Math. Ann. 254 (1980), 89-120.

8. J.-P. Serre, Problem 5359, Am. Math. Monthly 73 (1966), 89.

9. J. J. Sylvester, Mathematical question 11851, Educational Times 59 (1893), 98.

10. S. T. Yau, Calabi's conjecture and some new results in algebraic geometry, Proc. Nat. Acad. Sci. USA 74 (1977), 1798-1799.

Received September 20,1985. 